\title{
Catalyzing the Scale-Up of Crop Biofortification
}

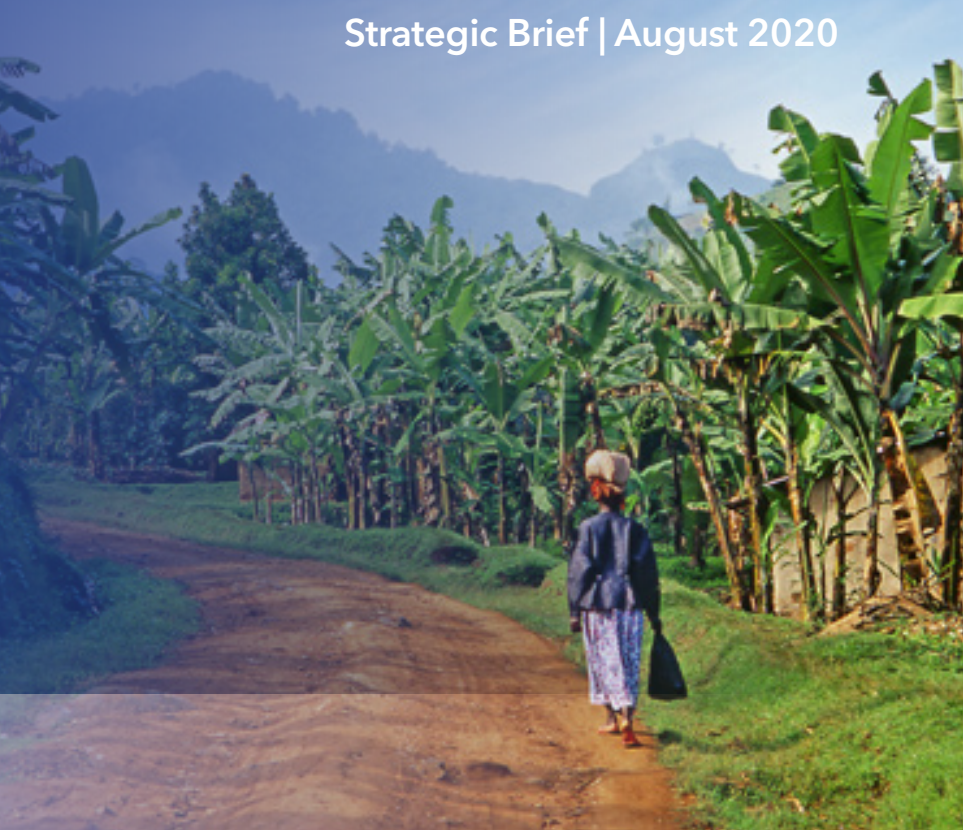

Arun Baral and Ekin Birol, HarvestPlus

Globally, an estimated two billion people suffer from micronutrient deficiencies that contribute to weakened immune systems, disease, disability, and even death. ${ }^{1}$ One of the main causes of micronutrient deficiencies - also known as hidden hunger - is low-quality diets that rely on calorie-rich but micronutrient-poor staple crops, and include very little nutrientdense foods such as animal-sourced foods and fresh fruits and vegetables.

Biofortification of staple crops is proven to be an efficacious, acceptable, cost-effective, and scalable solution for improving micronutrient intake, micronutrient deficiency status, and other health outcomes. Staple crops are the backbone of any food system; often one of the few crops grown by smallholder farmers, they are consumed, and are projected to be consumed, by all, regardless of demographics and socioeconomic status. ${ }^{2}$ Staples are also an equitable vehicle for improving the micronutrient intakes of women, adolescent girls, and children, who are most at risk of deficiencies due to their higher biological needs for key micronutrients, such as iron, zinc, and vitamin A, coupled with their limited access to micronutrient-rich foods, such as animal-sourced foods, often allocated to men or adolescent boys in their households. 3, 4, 6, 7

HarvestPlus, in close collaboration with crop breeding units of CGIAR Centers and National Agricultural Research Systems (NARS), facilitated the release of almost 370 conventionally-bred, high-yielding, climate-smart, and micronutrient-enriched varieties of 11 staple food crops in 41 countries, with hundreds of

1 Black, Robert E., et al, The Lancet, 2013.

2 Mango, Nelson, et al, Agriculture \& Food Security, 2018.

3 Branca, Francesco, et al, BMJ, 2015.

4 Ruel-Bergeron, Julie C., et al. PLoS One, 2015.

\section{Key Scaling Messages}

- Working with $600+$ partners globally since 2003 has enabled HarvestPlus to facilitate the release of almost 370 biofortified varieties of 11 staple crops in 41 countries, and delivery of biofortified seeds to $40+$ million people.

- Publishing on efficacy and effectiveness of biofortified crops in improving micronutrient status and health outcomes, establishing cost-effectiveness and return on investment of biofortification investments, and documenting lessons from delivery models implemented were critical to prepare biofortification for scaling up.

- To catalyze the scaling up of biofortification, HarvestPlus will (a) advise and monitor mainstreaming in CGIAR breeding programs, (b) enable production of biofortified foods along the value chain, (c) assist in embedding biofortification in national, regional, and multilateral programs and private investments, and (d) share its tools and learnings with value chain actors and interested stakeholders.

varietal lines being tested in these and in 20 more countries. Through HarvestPlus and its more than 600 partners' delivery efforts, an estimated 8.5 million farming households (translating to 42.4 million household members) had benefitted from biofortified crops by the end of 2019. HarvestPlus aims to contribute to transforming food systems to deliver healthy foods for all by catalyzing the development and scaling up of micronutrient-rich staple crops to benefit one billion people by 2030 .

5 De-Regil, Luz M., Kimberly B. Harding, and Marion L. Roche, The Journal of Nutrition, 2016.

6 Gittelsohn, J. and A.E. Vastine, The Journal of Nutrition, 2003.

7 Herrador Z, Perez-Formigo J, Sordo L, et al, PLoS One, 2015. 
In consultation with key partners, donors, and stakeholders, HarvestPlus developed a four-pronged strategy for rapid scale-up of biofortified foods:

1. Mainstreaming biofortification (i.e., breeding for micronutrient targets) in global (CGIAR) and national (NARS) breeding programs.

2. Catalyzing and enabling partnerships along the staple crop value chains to replace non-biofortified seeds, grains, ingredients, and foods with their biofortified counterparts.

3. Advocating to integrate biofortification in national, regional, and global policies, programs, and regulations to increase adoption and consumption of biofortified crops and foods, and to incentivize investments in biofortification by governments, private sector, civil society and, International Finance Institutions (IFIs), and international finance institutions (IFIs).

4. Coordinating a knowledge hub on biofortification interventions, and facilitating knowledge and best practices exchange among stakeholders and value chain actors for sustainable and cost-effective scale-up.

In parallel to its catalytic efforts to scale up biofortification, HarvestPlus will continue to invest in (a) targeted breeding to ensure a healthy pipeline of biofortified varieties are available until mainstreaming takes effect; (b) crop development research to assess the feasibility of developing new biofortified crops; (c) nutrition research on novel topics (such as biofortified crops and livestock health/productivity, noncommunicable diseases, gut microbiome); and (d) development, implementation, and evaluation of delivery models (especially in new geographies such as the Sahel), and documentation of lessons learned.

\section{Mainstreaming Biofortification}

A central tenet of a successful, long-term biofortification strategy is that all varietal lines co-developed by CGIAR Centers and NARS be biofortified. By piggybacking on the best agronomic characteristics of varieties coming out of agricultural research centers, currently grown varieties would eventually be replaced by higher-yielding biofortified varieties - thus "mainstreaming" these characteristics into all varietal lines.

Mainstreaming is easiest to accomplish for iron- and zincbiofortified crops, as increased micronutrient levels in the crops are invisible and tasteless to consumers in seeds/grain. Although mainstreaming will take time as all major breeding lines will have to be biofortified, this strategy is highly costeffective. Uptake does not depend on changing consumer behavior, and is often automatic and inevitable, relying on the profit-incentive of farmers. Success has been seen in production and consumption of zinc-biofortified rice and wheat in South Asia and iron-biofortified beans in East Africa. This strategy does not work as easily for vitamin A-biofortified crops, however, as increasing the density of vitamin A changes the grain's color to yellow or orange and can affect taste as well (albeit positively as evident from the literature). Demand must be generated for these varieties, involving raising consumer awareness. While widespread demand for these varieties is yet to be realized, color and taste are not barriers to consumer demand once household members taste the vitamin A varieties and understand the reason for the color change - and that improving vitamin A intake with the new variety does not increase the cost.

Investing in "targeted" breeding programs at CGIAR Centers and NARS was necessary to prove the concept of biofortification. Doing so not only proved high yields could be combined with mineral and vitamin density, but also built crop development pipelines.

Facilitating the release and delivering the seeds of biofortified varieties in several countries across Africa, Asia, and Latin America over the past 15 years provided experience and learnings in different delivery models along the value chain. With this crop development and delivery success, positive nutrition and impact evidence generated, and a successful global advocacy campaign in place, biofortification is now generally well accepted in the international development and nutrition communities as an efficacious and cost-effective intervention for addressing micronutrient deficiencies and improving diet quality. The time has come to start transitioning out of targeted breeding for biofortification, and to move to mainstreaming of nutritional traits in broader germplasm of major staple crops. Biofortification mainstreaming would help address micronutrient needs of billions of people whose diets are based on these staples, sustainably and cost-effectively.

Additional, direct funding to CGIAR Centers for mainstream breeding of iron and zinc varieties will be an integral part the CGIAR's Excellence in Breeding (EiB) Platform. As approval is secured for direct funding, CIMMYT, ICRISAT, and IRRI will be the first to demonstrate how to transition from targeted breeding to mainstreaming biofortified traits into the best parental lines. ${ }^{8}$ HarvestPlus will continue to provide advice and guidance in the development of the mainstreaming workplans, monitoring of outputs and outcomes, and use evidence and advocacy to influence accountability to mainstream biofortified traits.

Achieving and coordinating mainstreaming goals across crops and food systems requires a holistic approach - especially in the areas of resource mobilization, micronutrient target setting, 
quality control, monitoring, standards, advocacy, and public support.

\section{Catalyzing and Enabling Partnerships}

The challenge and opportunity of scaling requires catalyzing and enabling partnerships along the biofortification value chain to increase the production and consumption of biofortified foods. Effective partnerships with public, private, and civic, nonprofit and humanitarian entities, of all sizes and scales, will strengthen part-

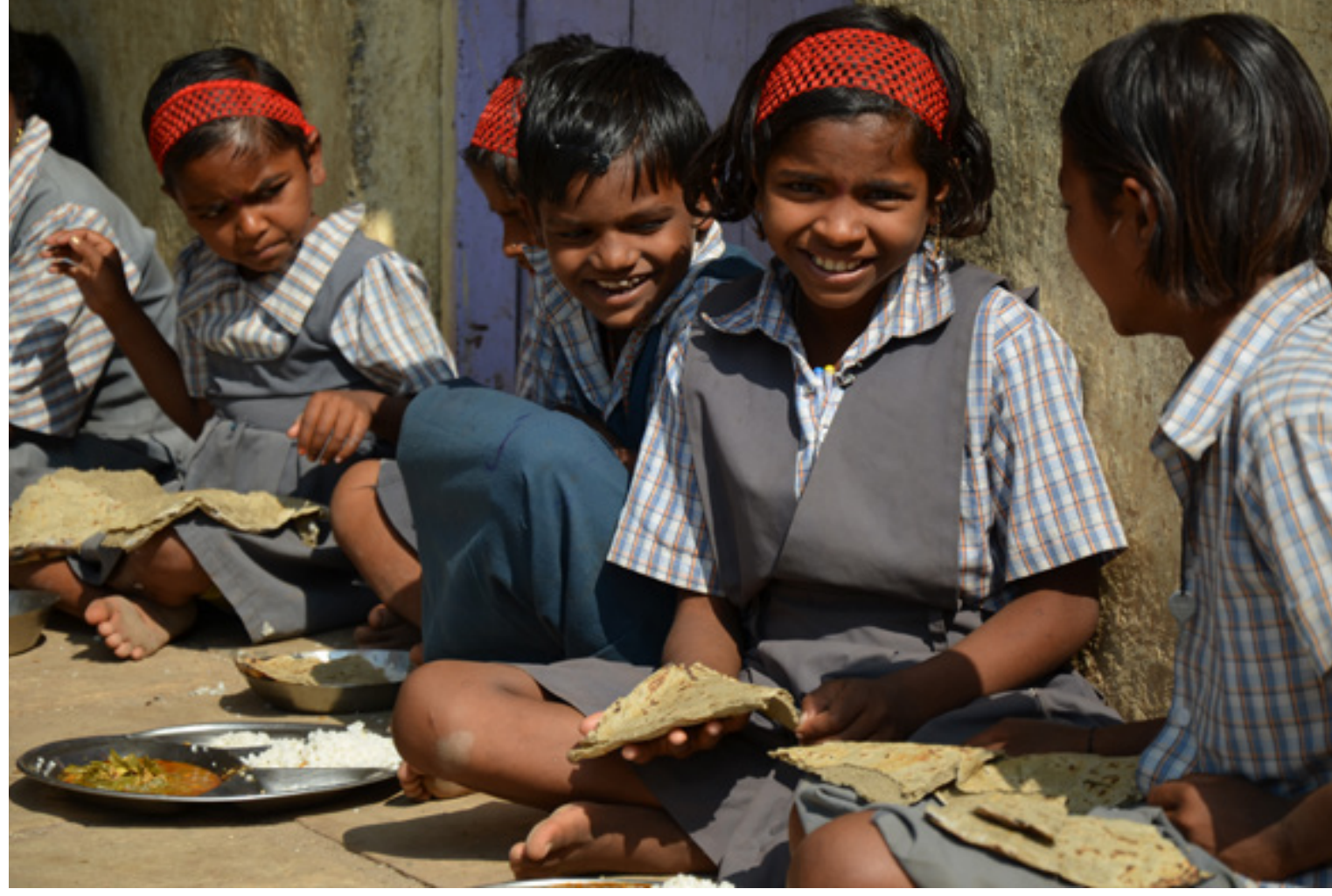
ners' capacities to cost-effec-

tively or profitably and sustainably integrate biofortification in their operations, programs, and investments.

Crop research and development: In addition to CGIAR Centers mainstreaming biofortification in breeding programs, selected NARS will need continued support to strengthen capacity and improve skills and knowledge to carry out biofortified breeding. Capacity development at crop trait research and crop development stages will include training public sector breeders (NARS) and the private sector, mostly small and medium-sized seed companies. Trainings will include improved methods in biofortification breeding; using diagnostic tools; identifying best germplasm; selecting the best breeding environments; and micronutrient bioavailability and retention. HarvestPlus has fostered the development of independently funded biofortification programs with large research portfolios in Brazil, China, and India, and will continue its current collaborations with these three programs, which are then expected to influence the development of biofortified crops in additional countries.

Seed multiplication: Partnerships with private seed companies are particularly important for hybrid crops such as maize in Africa (e.g., SeedCo) and pearl millet in India (e.g., Nirmal Seeds). HarvestPlus has also worked with private wheat and rice seed companies in India and Bangladesh, respectively. Experience has shown smaller companies are more readily inclined to become involved with biofortified crops to increase market share, while larger companies enter when demand is already established. HarvestPlus will continue to engage with private seed companies by providing them with information/ training on the benefits of biofortification and quality control, and access to biofortified lines - where needed - for integration of biofortified lines in their portfolios. For crops or seed systems that don't lend themselves to investments by the private sector, public sector multipliers, NGOs, women's groups, and farmer organizations will continue to be targeted for trainings on producing high-quality seeds to grow biofortified crops.

Distribution to and demand creation for farmers: Farmers are critical to successfully developing and adopting biofortified varieties. Their engagement must continue, not only in codevelopment and testing of new varieties, but also to develop their capacity in nutrition habits, improved agronomy practices, crop rotation, pest management, and using digital technologies to access markets. HarvestPlus works in partnership with public, private, and civil society/NGO sectors to carry out these activities.

Food processing and value addition: Partnerships with small and medium-sized food processing and manufacturing companies, including InnerBeing in India, GraceCo in Nigeria, PRAN foods in Bangladesh, and Sky Brand in Zimbabwe, have generated learning and experience. Future training should cover developing healthy foods using biofortified ingredients; retaining micronutrients during processing, packaging, transportation, and storage; maintaining traceability (i.e., supply chain integrity); and food safety, as well as capacity development in marketing, contract development, nutrition labelling, and accessing finance.

Retailers and consumers: According to acceptance studies conducted among rural and urban consumers, traditional foods made with biofortified crops are liked as much as - if not more than - the same foods made with non-biofortified crops, sometimes even in the absence of nutritional benefit information. 
Awareness-raising and demand-creation campaigns targeting a wide range of consumers and a partnership with the Global Alliance for Improved Nutrition (GAIN) on commercialization are helping to build a knowledge base on efficacious, cost-effective, scalable, and sustainable media and messaging to engender consumer demand for biofortified foods. Engagement with leading global food manufacturers and retailers creates global awareness and demand for "naturally-nutritious" biofortified products, as demand "pull" mechanisms.

\section{Advocating for Policy and Program Integration}

To ensure sustainability of biofortification interventions implemented to date, it is crucial to ensure biofortification is included in the policies, strategies, and plans of national governments, IFIs, and UN agencies; resources are then allocated to implement these policies, strategies, and plans.

To date, 24 countries have included biofortification in their national agricultural and/or nutrition agendas, policies, plans, and programs: Bangladesh, Burkina Faso, Burundi, Colombia, Democratic Republic of Congo, El Salvador, Ethiopia, Ghana, Guatemala, Honduras, India, Kenya, Malawi, Mozambique, Nicaragua, Nigeria, Pakistan, Panama, Rwanda, Senegal, Tanzania, Uganda, Zambia, and Zimbabwe. In November 2019, agriculture ministers of the African Union (AU) recommended to their heads of state officially endorsing biofortification. At the international level, UN and Rome-based agencies have integrated biofortification in programs and recommendations, including UNICEF's State of the World's Children 2019 Report, World Food Programme's local and regional food procurement policy, and IFAD's Nutrition Sensitive Value Chains guidelines. Several IFIs, including the World Bank, African Development Bank and Inter-American Development Bank, have included biofortification in nutrition-sensitive agricultural investment plans and strategies, and the World Bank has issued grants and loans that include biofortification. Work will continue with these and others, including the Asian Development Bank and the Islamic Development Bank, to integrate biofortification in plans, strategies and grants; and to evaluate the impact, and conduct cost-benefit analyses of investments.
Future policy and advocacy efforts will include developing evidence on nutritional efficacy and cost-benefit/cost-effectiveness analysis and return on investment of including biofortification in specific policy and regulatory changes and public programs, such as subsidy or safety net programs. Such information is expected to catalyze national governments and the private sector to invest more of their own resources to incorporate biofortified seeds and foods into their programs, reducing the need for international aid for food and nutrition security.

To strengthen the global enabling environment, HarvestPlus will continue to engage with and provide evidence to the WHO and the FAO Review Committee to facilitate their issuance of the guidance to UN member states' health and agriculture ministries and provide input to the UN Committee on World Food Security Voluntary Guidelines on Food Systems and Nutrition. Considerable progress toward a global definition for biofortification was achieved through the FAO/WHO Codex Alimentarius standard-setting process, and publicly available standards are currently being developed for all biofortified crops.

\section{Biofortification Knowledge \& Information Hub}

One main pillar of the catalyzing biofortification scale-up strategy is to produce and share all evidence, learnings and tools with other stakeholders, empowering them to implement and scale biofortification interventions on their own. To achieve this, HarvestPlus is developing a Knowledge \& Information Hub with tools to help estimate effective breeding targets for each crop-micronutrient-country combination; cost-effectiveness and cost-benefit analyses of planned, ongoing, and completed biofortification interventions and tools to better target interventions across and within countries; documents synthesizing evidence generated to date; an electronic, searchable library of biofortification products; guidelines on how to introduce, scale-up, and anchor sustainable and cost-effective biofortification interventions; and monitoring, evaluation, learning, and impact assessment guidance. The Hub will facilitate knowledge exchange and learning among stakeholders.

Arun Baral is Chief Executive Officer and Ekin Birol is Director of Impact and Strategy for HarvestPlus. Learn more at www. harvestplus.org.
INTERNATIONAL FOOD POLICY RESEARCH INSTITUTE

A world free of hunger and malnutrition

IFPRI is a CGIAR Research Center

1201 Eye Street, NW, Washington, DC 20005 USA

T. +1-202-862-5600 | F. +1-202-862-5606 | ifpri@cgiar.org | www.ifpri.org

Copyright @ 2020 International Food Policy Research Institute. All rights reserved. Contact ifpri-copyright@cgiar.org for permission to republish.

Photo credits: Pecold/Shutterstock; Alina Paul-Bossuet.
VISIT A4NH ONLINE www.a4nh.cgiar.org

$\square$ CONTACT A4NH John McDermott A4NH Director j.mcdermott@cgiar.org
TWITTER @A4NH_CGIAR

HarvestPlus leads A4NH's Biofortification flagship. To learn more, visit www.a4nh.org 